\title{
Interference with spatial alternation by exposure to other mazes
}

\author{
W. SCOTT TERRY \\ University of North Carolina at Charlotte, Charlotte, North Carolina
}

\begin{abstract}
Rats were trained in a spatial alternation task in an E-shaped maze, and then were tested for retention over 60 -sec delays. The subjects received an interfering alternation trial during the delay interval in one of two other mazes: either a T-maze, or a parallel $(\mathrm{P})$ maze with adjacent goal arms. The T-maze produced more alternation than did the P-maze. The main findings were that (1) the T-maze trial produced interference in retention of the target maze, and (2) the P-maze produced interference in retention of the target maze only when an error had occurred in the P-maze. These results suggest that interference occurs when the distractor event itself places demands on the processing resources of the subject, and thus competes with processing of the target memory.
\end{abstract}

Retroactive interference (RI) of working memory is defined as the interpolation of a distractor event between the occurrence of a target stimulus and the test of memory of the target. Spatial memory of rats in the radial maze has been shown to be relatively resistant to RI (Maki, Brokofsky, \& Berg, 1979). Roberts (1981) was only able to produce RI by using extensive distractor treatments (i.e., exposure to three other mazes during the retention interval). Retention in the radial-arm maze is typically robust, whether defined by duration, capacity, or resistance to interference. The purpose of the present study was to determine whether spatial memory as tested in another maze task would prove more susceptible to interference.

Miller, Greco, Marlin, and Balaz (1985) found RI in three delayed-alternation experiments, using as a distractor a holding box containing other rats. A fourth experiment showed interference when the rats were exposed to a portion of the to-be-remembered maze. Miller et al. (1985) suggested that interference could occur either due to the similarity of the distractor to the target, or due to interference in the cognitive processing necessary to retain the target representation in memory.

The rationale for the present manipulation was to use a distractor that would draw on the same cognitive resources as the main task - that is, short-term retentionwhile also varying the similarity and difficulty of the distractor task. The present study contained alternation trials in one of two other mazes as interfering events. One distractor was a T-maze, which required the same left-right alternation as the target maze. The other distractor was a modified Grice box, which has goalboxes parallel and adjacent to one another. The T-maze has been shown to

Correspondence may be addressed to W. Scott Terry, Department of Psychology, University of North Carolina at Charlotte, Charlotte, NC 28223 . produce more spontaneous (Douglas, Mitchell, \& Del Valle, 1974) and trained (Roberts, 1972) alternation than the parallel maze, presumably because the latter produces less discriminable memories of the two goal locations. Thus, the possible sources of interference in the present study were increased, either because of the similarity of the T-maze to the target maze, or because of the greater difficulty of the parallel-maze task.

\section{METHOD}

\section{Subjects}

The subjects were 12 male Harlan Sprague-Dawley rats. The animals were 100 days old at the start of the experiment.

\section{Apparatus}

The main maze was E-shaped, having the center leg as the startbox and the two outside legs as goalboxes. One distractor maze was T-shaped. The E- and T-maze dimensions are described in detail in Terry and Anthony (1980). The second distractor apparatus, the parallel maze (P), was basically a large rectangle, divided by sliding doors into a startbox $(30 \times 20 \mathrm{~cm})$, choice area $(60 \times 20 \mathrm{~cm})$, and goalboxes (each $28 \times 10 \mathrm{~cm})$ All mazes were made of plywood and painted black, uncovered. They differed in alley width, side height, and floor material. The E-maze was placed on a table facing a blank wall, with the legs of the maze pointing toward the center of the room and the experimenter. The other two mazes were placed adjacently on a table opposite the main maze, also facing a wall. The startboxes of the target maze and of the distractor mazes were about $1 \mathrm{~m}$ apart. Thus, the experimenter simply had to rotate $180^{\circ}$ to take an animal from the target maze to the distractor maze, and vice versa.

\section{Procedure}

The subjects were maintained on a 22-h food-deprivation schedule, with water available ad lib except during the experimental sessions. The subjects received four daily periods of free exploration in the E-maze, followed by six sessions of nondelay alternation training. On the first run of each alternation trial, each subject was forced to enter one of the goal arms, the other arm being blocked at the choice point. After consuming the food reward, the animal was returned to the startbox, a 3-sec delay was timed, and the animal was released from the startbox. The subject was now free to enter either goal, but food was only available in the goal opposite that entered on the forced-choice run. In case of error, free-choice runs were repeated until correct alternation 
occurred. The correct choice varied over trials in a sequence that equated the number of left and right turns daily, and balanced left-right transitions between trials. During the last session, the animals were allowed to explore the other two mazes that were to be used as distractors.

The experimental phase followed next. Each session consisted of four experimental trials with delay intervals between the forced- and freechoice runs in the E-maze. Two of these trials were control trials, in which the animals were simply detained in the holding cage for the 60 sec retention interval. The other two trials were distractor trials, in which the subjects received an alternation trial in either the T-maze or the Pmaze during this interval. In each case, the subjects were given a reinforced forced-choice run to one goal and then were given an immediate free-choice run with food available in the opposite goal. Following the distractor-maze runs, the animal was returned to the holding cage to finish out the 60-sec delay. Then the subject received its free-choice trial in the target E-maze. The sequence of control and interference trials was balanced across subjects within sessions, and within subjects between sessions.

The subjects received six sessions of testing as described above. In addition, a seventh session was given with only the P-maze as a distractor. The maze was rotated $90^{\circ}$ for this session, to control for direction of movement during the RI manipulation. The subjects ran from left to right on two trials, and right to left on the other two.

Four additional sessions were conducted to assess the effects of a Tor P-maze trial prior to the target-maze trial. The data from this "proactive interference" manipulation will not be described further, other than to provide base accuracy rates for the distractor mazes when not embedded within the E-maze trials. These sessions occurred between Days 4 and 5 of the RI series.

Each session began with a no-delay warmup trial in the E-maze. Rewards consisted of three Noyes pellets in the E-maze goal, and two pellets in the distractor-maze goals. In cases of error in any of the mazes, the subjects were allowed to retrace to the correct goal. Intertrial intervals averaged $5 \mathrm{~min}$. Additional details of procedure may be found in Terry (1987).

\section{RESULTS}

The data are reported in terms of the mean percentages of correct choices (alternations) on free-choice runs. Statistical significance is defined as $p<.05$.

The results are shown in Table 1 . The top row shows the major data of interest, performance on the target maze as a function of the RI manipulation. These trials all had a 60-sec retention interval. As can be seen, on trials with an interpolated $\mathrm{T}$-maze alternation trial there was a large decrement in performance. Interpolation of a parallelmaze trial had a smaller decremental effect on E-maze retention. A simple analysis of variance, comparing within-subjects performance on the three trial types, showed a significant overall effect $[F(2,22)=4.39]$. Newman-Keuls tests showed there was significantly less accuracy in the T-maze RI condition than in the control condition. The P-maze RI condition was only marginally

Table 1

Mean Percentage Correct Alternation in the Target and (E) and Distractor ( $T$ and P) Mazes on Retroactive Inhibition (RI) Trials and on Control Trials

\begin{tabular}{cccc}
\hline & \multicolumn{3}{c}{ RI Conditions } \\
\cline { 2 - 4 } Maze Tested & Control & T-Maze & P-Maze \\
\hline Target Maze & 78.20 & 59.75 & 69.50 \\
Distractor-T & 95.20 & 72.20 & \\
Distractor-P & 43.75 & & 51.30 \\
\hline
\end{tabular}

significantly different from each of the other two conditions, $p<.10$.

Rotating the parallel maze $90^{\circ}$ had no effect. There were $68.75 \%$ correct choices during this session, a value comparable to that for the nonrotated maze.

The next two rows of Table 1 show performance on the distractor mazes, which had no delay interval between free- and forced-choice runs. Alternation was significantly better in the T-maze than in the P-maze during the RI phase $[t(11)=2.32]$. This finding is consistent with previous reports of the difficulty in training alternation in a Grice box (Roberts, 1972). Accuracy in the parallel maze showed no improvement with training, averaging just under $50 \%$ throughout the study. Alternation in the T-maze occurred immediately in the RI phase, and was maintained at the same level, about $70 \%$, throughout the experiment. However, embedding the T-maze trial in the retention interval of the target-maze trial did have a detrimental effect on distractor-maze performance. As can be seen in Table 1, performance on T-maze trials remote from other trials averaged around $96 \%$. This difference is highly significant $[t(11)=3.33]$. Thus, in this RI condition, there was mutual interference: the T-maze interfered with performance in the target maze, and vice versa.

Further analysis of the P-maze trials showed a difference between correct and incorrect trials. When a correct choice occurred in the P-maze, there was greater likelihood of a correct alternation in the target maze (72.6\%). When an error occurred in the P-maze, then alternation dropped $(61.3 \%)$. This difference was significant $[t(11)=2.72]$. Although the subjects were allowed to correct errors, the momentary frustration of not finding food may have been sufficient to disrupt memory for the target location. A similar effect did not occur with the T-maze, although the higher accuracy in this maze provided only a small number of error trials for analysis.

\section{DISCUSSION}

The basic finding in this study was that retroactive interference could be obtained in a spatial-maze task. The data showed that a T-maze interference event reduced retention, whereas a parallel-maze trial had a smaller effect. Both distractor trial types share some similarities: both involve food reward, handling, and exposure to alternate spatial locations during the delay. Thus, the difference in effectiveness of the $T$ and parallel mazes must lie elsewhere.

There are two possible reasons for greater T-maze interference. It is possible that the T-maze was more similar to the target maze, thus causing more interference due to confusion of memory traces between the two mazes. Although Roberts (1981) found little evidence for similarity interference in a radial maze, Miller, Greco, Marlin, and Balaz (1985) did find RI in a T-maze task. Similarity based on direction of movement, a factor previously found to affect alternation (Sherrick, Brummer, Roth, \& Dember, 1979), was tested here, but it was not found to affect interference.

A second interpretation is based on Miller et al.'s (1985) suggestion concerning processing interference: interference can occur because the limited-capacity memory system becomes overloaded. In the present case, although the parallel maze was supposed to be more difficult, it may not in fact have imposed on the subject's working memory. The subjects were performing at chance levels even after a zero-second delay. Thus, there is no evidence for active retention that could have 
interfered with the target-maze memory. In the T-maze, on the other hand, the subjects performed fairly accurately, thus indicating the use of both working memory (to choose the correct goal) and reference memory (for the alternation rule) during the retention interval. Such processing may have more effectively interfered with retention of the target-maze location.

The present results add to what is known of other sources of processing interference, such as exposure of subjects to multiple to-beremembered mazes (Roberts, 1981) or to surprising stimuli during the delay (Miller \& Balaz, 1983). In contrast, Terry (1987) found that simply giving a food reward during the delay interval did not adversely affect retention. This may have been because the feeding did not impose any processing demands.

Terry and McSwain (1984) found that the surprising absence of food enhanced retention of the nonrewarded goal. This suggests that a frustration event may engage memory processing. The present findings with a P-maze distractor provide converging evidence for this notion. In the present case, however, the engaging of processing during the retention interval distracted processing from the target memory.

\section{REFERENCES}

Douglas, R. J., Mitchell, D., \& Del Valle, R. (1974). Angle between choice alleys as a critical factor in spontaneous alternation. Animal Learning \& Behavior, 2, 218-220.

MAKI, W. S., Brokofsky, S., \& BerG, B. (1979). Spatial memory in rats: Resistance to retroactive interference. Animal Learning \& Behavior, 7, 25-30.
Miller, R. R., \& Balaz, M. A. (1983). Postacquisition unexpected footshock disrupts appetitively motivated instrumental performance based on short-term retention. Bulletin of the Psychonomic Society, 21, 225-228.

Miller, R. R., Greco, C., Marlin, N. A., \& Balaz, M. A. (1985). Retroactive interference in rats: Independent effects of time and similarity of the intervening event with respect to acquisition. Quarterly Journal of Experimental Psychology, 37B, 81-100.

ROBERTS, W. A. (1972). Spatial separation and visual differentiation of cues as factors influencing short-term memory in the rat. Journal of Comparative \& Physiological Psychology, 78, 284-291.

ROBERTS, W. A. (1981). Retroactive inhibition in rat spatial memory. Animal Learning \& Behavior, 9, 566-574.

Sherrick, M. F., Brunner, R. L., Roth, T. G., \& Dember, W. N. (1979). Rats' sensitivity to their direction of movement and spontaneous alternation behavior. Quarterly Journal of Experimental Psychology, 31, 83-93.

TERRY, W. S. (1987). Food priming and short-term retention in the delayed alternation task. Learning \& Motivation, 18, 261-273.

TERRY, W. S., \& ANThONY, S. G. (1980). Arousal and short-term memory: Effects of caffeine and trial spacing on delayed-alternation performance. Animal Learning \& Behavior, 8, 368-374.

TERRY, W. S., \& McSwain, B. J. (1984). Effects of reward amount and reward omission on short-term retention. Animal Learning \& Behavior, 12, 62-68.

(Manuscript received September 23, 1988.) 